\title{
Aspectos históricos e metodológicos da medição e estimativa da taxa metabólica basal: uma revisão da literatura
}

\author{
Historical and methodological aspects \\ of the measurement and prediction of basal \\ metabolic rate: a review
}

Vivian Wahrlich 1

Luiz Antonio dos Anjos 1,2

\footnotetext{
${ }^{1}$ Centro de Estudos da Saúde do Trabalhador e Ecologia Humana, Escola Nacional de Saúde Pública, Fundação Oswaldo Cruz. Rua Leopoldo Bulhões 1480, Rio de Janeiro, RJ 21041-210, Brasil. wahrlich@ensp.fiocruz.br 2 Laboratório de Avaliação Nutricional e Funcional, Departamento de Nutrição Social, Universidade Federal Fluminense. Rua São Paulo 30 4o andar, Niterói, RJ 24040-115, Brasil. anjos@ensp.fiocruz.br
}

Abstract This paper reviews the most relevant aspects of measuring basal metabolic rate (BMR). The authors discuss individual and lifestyle factors (age, anthropometry, smoking, physical activity, diet, menses, and ethnicity) as well as possible environmental factors at ti me of measurement which may alter BMR values. Vari ous available predictive equations, especially those recommended by the World Health Organization in 1985, and subsequent validation studies, particularly in populations living in the tropics, are presented. The review indicates the need for more information on BMR from populationsliving in different parts of the world so that better estimates can be provided for clinicians and epidemiol ogists.

Key words Anthropometry; Measurements; Nutrition Assessment

Resumo A taxa metabólica basal (TMB) é uma das informações fisiológicas mais importantes em estudos nutri cionais clíni cos ou epidemiológi cos, seja para se determinar as necessidades energéticas ou calcular o gasto energético de indivíduos ou populações. O presente arti go di scute, com basenuma revisão da literatura, a i mportância e os aspectos mais rel evantes da medi ção da TMB. Inicialmente, faz-se um histórico do desenvolvimento dos conceitos e da técnica de medição da TMB até os dias atuais. São discutidos os fatores indivi duais (idade, antropometria, tabagismo, atividade física, dieta, ciclo menstrual e etnia) eos possíveis fatores ambientais presentes na hora da medi ção (temperatura, local e repouso) que podem influir no valor da TMB. Apre senta-se as várias equações de predi ção de TMB di sponíveis, em especial a sugeri da pela Organização Mundial da Saúde em 1985, e di scute-se os estudos real izados desde então, que tentam validar seu uso internacional mente, particularmente, em populações vivendo nos trópi cos. O artigo aponta para a necessi dade dese obter mai s informações sobre o metabolismo basal nos vários segmentos populacionais vivendo em diferentes regiões do mundo, para que mel hores estimativas possam ser forneci das tanto para o uso dínico quanto epidemiológico.

Palavras-chave Antropometria; Medições; Avaliação Nutricional 
Introdução

A taxa metabólica basal (TMB) é a quantidade de energia necessária para a manutenção das funções vitais do organismo, sendo medida em condições padrão de jejum, repouso físico e mental em ambiente tranqüilo com controle de temperatura, iluminação e sem ruído (Bursztein et al., 1989; Garrow, 1974; Harris \& Benedict, 1919). Desde o século XIX, a medição da TMB é feita através da determinação da quantidade de cal or produzida pelo organismo (calorimetria direta) ou pelo cálculo de calor indiretamente (calorimetria indireta) a partir do consumo de oxigênio $\left(\dot{\mathrm{V}}_{2}\right)$ e excreção de gás carbônico $\left(\dot{\mathrm{V}} \mathrm{CO}_{2}\right)$ tanto para fins diagnósticos quanto nutricionais. Entretanto, somente a partir do estudo de Harris \& Benedict, em 1919, é que houve uma tentativa de sistematização das informações existentes sobre o metabolismo basal com o desenvolvimento de equações de predição da TMB, a partir de medidas antropométricas, já que a calorimetria não era muito disponível. Com a mudança de orientação na estimativa das necessidades energéticas humanas, da ingestão para o gasto energético sugerida pela Food and Agriculture Organization/World Health Organization/United Nations University (FAO/WHO/UNU, 1985) houve a necessidade de atual ização das informações existentes sobre o metabolismo basal, com revisão das equações de predição da TMB (Schofield, 1985). A partir de então, vários estudos têm demonstrado que as equações tendem, em sua maioria, a superestimar a TMB em várias populações, principalmente, as que vivem nos trópicos (Henry \& Rees, 1991). O objetivo do presente artigo é revisar vários aspectos relacionados à medição da TMB, incluindo al guns aspectos históricos, e a validade do uso internacional, particularmente nas populações vivendo nos trópicos, das equações de predição atualmente existentes.

\section{Importância da medida de TM B}

ATMB éo principal componente do gasto energético diário, podendo representar de $50 \%$ (nos indivíduos muito ativos fisicamente) a 70\% (nos mais sedentários) do total de energia gasta diariamente (Clark \& Hoffer, 1991). Baseado nesta evidência, o gasto energético (GE) diário deveria nortear necessariamente as recomendações energéticas. Apesar de ser uma conclusão óbvia, somente a partir de 1985 que se passou a proceder desta forma (FAO/WHO/UNU, 1985). Até então, as recomendações energéticas po- pulacionais eram derivadas de informações sobre o consumo alimentar de populações, ditas saudáveis (Schoeller, 1999) valores que, nor-

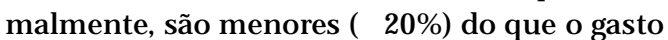
energético medido por água duplamente marcada (Seale \& Rumpler, 1997). Nesta nova orientação os componentes do gasto energético deveriam ser expressos como múltiplos da TMB, numa tentativa de se controlar as características individuais como as dimensões e composição corporais, o estado nutricional e características demográficas como idade e gênero. Como se reconhecia que não havia uma grande disponibilidade de calorímetros, os órgãos internacionais (FAO/WHO/UNU, 1985) sugeriram o uso de equações de predição para estimar aTMB. Assim, tanto para o nível individual quanto o populacional, a TMB é o valor base para se estabelecer as necessidades energéticas (James \& Schofield, 1990).

O uso da TMB no estabelecimento das necessidades energéticas, por si só, já seria motivo suficiente para sua determin ação nos vários segmentos da população da forma mais exata possível, seja em avaliações clínicas ou epidemiológicas. Além disso, seu uso em estudos epidemiológicos sobre a ingestão alimentar e a determinação do nível de atividade física, também demonstra sua importância. Por exemplo, usualmente, avalia-se o grau de confiabilidade na informação sobre a ingestão energética de indivíduos dividindo-se o valor de energia ingerida, pelaTMB. Quando em pessoas obesas, o valor desta razão é menor do que 1,2 costuma-se dizer que os indivíduos subestimaram a ingestão, visto que, os mesmos não poderiam ser obesos ingerindo somente 1,2 vezes a TM $B$ (MacDiarmid \& Blundell, 1997) valor que é considerado como de manutenção (James \& Schofield, 1990). Na análise da dieta da população americana com idade superior a vinte anos, investigada através de recordatório de 24h durante a fase I do estudo americano NHANES III (National Health and Nutrition Examination Survey) $(n=7.769)$ e com o valor deTM $B$ estimado através de equações de predição, o valor médio da razão no total das mulheres foi de 1,26, sendo de 1,09 nas obesas (Briefel et al., 1995). Anal isando o mesmo banco de dados, Briefel et al. (1997) utilizaram o valor de 0,9 como critério para subestimativa de ingestão, encontran do $18 \%$ de homens e $28 \%$ de mulheres na população americana abaixo deste valor. Entre estes, havia mais indivíduos com sobrepeso e sedentários do que os que tinham valores superiores a 0,9. Duas possibilidades de explicação para estes valores emergem. Inicialmente, deve-se questionar o método de obten- 
ção da informação de ingestão alimentar, seja através de recordatório de $24 \mathrm{~h}$ ou por registro (diário) alimentar, visto que os indivíduos, principalmente os obesos (Prentice et al., 1996), tendem a subestimar a ingestão, quando são obrigados a dar tal informação. Ou seja, o numerador da razão pode estar subestimado. Por outro lado, também é possível que a TMB (o denominador) esteja sendo superestimado pelas equações de predição, mesmo na população americana (Clark \& Hoffer, 1991; Owen et al., 1986, 1987). Assim, as conclusões sobre o estado nutricional de populações (e dos indivíduos) padecem de credibilidade, em parte, pela não mensuração da TMB.

Da mesma forma, o nível de atividade física (NAF), representando a razão entre o gasto energético e a TMB de $24 \mathrm{~h}$, é utilizado com relativa freqüência, como um índice na classificação da atividade física de indivíduos em estudos epidemiológicos (Anjos, 1999). Alguns autores utilizam o valor do NAF leve $(1,55$ e 1,56 , para homens e mulheres, respectivamente; $\mathrm{FAO} / \mathrm{WHO}$ / UNU, 1985) como valor abaixo do qual se caracterizaria indi víduos como sedentários (Cervato et al., 1997; Martins et al., 1993; Rego et al., 1990). Deve-se ter em mente que esses valores foram recomendados para se calcular as necessidades energéticas, baseando-se nas características ocupacionais da população. Como na maioria das situações clínicas ou dos estudos epidemiológicos não se mede a TMB, deve-se confiar em equações de predição daTMB, o que em muitos casos, pode levar a conclusões errôneas ( Wahrlich \& Anjos, 2000). Por exemplo, Vinken et al . (1999) encontraram valores de NAF médio de 1,80 para 93 indivíduos (44 homens e 49 mulheres) de 18 a 81 anos de idade, ao medir o gasto energético de $24 \mathrm{~h}$ através de água duplamente marcada (método atualmente preferido para sua medição; Schoeller, 1999) e a TMB por cal orimetria indireta. Os autores concluíram que os valores de NAF recomendados deveriam ser revistos, opinião que não é consensual para os países em desenvolvimento (Sheety et al., 1996). Black et al. (1996) revisaram as informações de 574 medições de gasto energético através de água duplamente marcada e encontraram valores médios de NAF, semel hantes aos sugeridos pel o Comitê de Expertos da FAO/WHO/UNU (1985) o que fez com que os autores sugerissem a manutenção dos valores de NAF. Da mesma forma, os autores sugerem a manutenção de valores recomendados pelo Departamento de Saúde do Reino Unido, que incorporam as atividades não ocupacionais e que são, em geral, menores do que os da FAO/WHO/UNU (1985) principalmente para as atividades pesadas. Para as populações vivendo em condições menos desenvolvidas, não existem dados confiáveis suficientes de gasto energético, mas parece ser necessário revisar os valores de NAF, baseado nas evidências de que, aparentemente, os dados de gasto energético (numerador) usados para seu cálculo foram subestimados (Haggarty et al., 1994; Heini et al., 1996) além da evidente superestimativa da TMB (denominador) usada para essas populações (Cruz et al., 1999; Henry \& Rees, 1991; Wahrlich, 2000). De qualquer jeito, e para qualquer uso que se tenha em mente, a TMB deve ser obtida da forma mais exata possível.

\section{Aspectos históricos do estudo}

do metabolismo basal

O interesse sobre o metabol ismo remonta à antigüidade, quando surgiu a teoria da "perspiração insensível" da escola de Hipócrates (King, 1924) que baseava-se na observação de que um adulto não ganhava peso apesar de ingerir uma quantidade de alimento e líquido muito superior a sua excreção. Foi somente em 1780 que o metabolismo começou a ser melhor entendido através dos experimentos de Antoine Laurent Lavoisier (Lusk, 1917) nobre francês que identificou que um gás, por ele chamado de oxigê nio, mas anteriormente denominado "ar de fogo" (dephlogisticated air) por Joseph Priestley (McHenry \& Beaton, 1963), combinava-se com substâncias combustíveis liberando calor. Para provar que a oxidação era a fonte de calor em animais, Lavoisier construiu, juntamente com Laplace, o primeiro calorímetro direto para animais e desenvolveu o princípio da calorimetria indireta ao medir a quantidade de gás carbônico produzida pelo animal durante o mesmo período da calorimetria direta (Taylor et al., 1956). Nesse experimento, Lavoisier conseguiu evidenciar que a quantidade de calor produzida pelo animal era praticamente igual ao calor cal culado a partir do gás carbônico produzido pela respiração. Com isto, ficou comprovada a hipótese de que o carbono contido no organismo combinava-se com o oxigênio para produzir calor. Lavoisier estendeu seus experimentos para o homem, estudando aparentemente, apenas as trocas gasosas e demonstrando que a exposição ao frio, a digestão e a atividade física eram fatores que elevavam o consumo de oxigênio (Du Bois, 1936; King, 1924) e, conseqüentemente, a produção de calor ou metabolismo.

Durante o século XIX, com o estabelecimento das leis da termodinâmica, vários calo- 
rímetros diretos e câmaras respiratórias foram desenvolvidos (Durnin \& Passmore, 1967) na tentativa de aperfeiçoar as técnicas propostas por Lavoisier. Neste período, também ocorreram descobertas i mportantes que possibilitaram o desenvolvimento de métodos mais exatos para medir o metabolismo através das trocas gasosas, como a identificação dos substratos oxidados pelo organismo, a quantidade de oxigênio necessária para oxi dá-los e o valor calorífico dos componentes dos alimentos quando oxidados no organismo (Lusk, 1917; Mchenry \& Beaton, 1963)

Em 1894, Rubner construiu o primeiro caIorímetro direto de êxito para estudo experimental em cães (Webb, 1985). Em conexão ao calorímetro, foi utilizado o aparelho de Pettenkofer-Voit que possibilitava, além de medir a perda de calor, obter a quantidade de gás carbônico produzida. Neste caso, ele utilizava ao mesmo tempo a calorimetria direta e indireta, podendo observar a concordância entre os valores obtidos pelos dois métodos (Boothby \& Sandiford, 1920) e, provando assim, que a lei de conservação de energia também se aplicava aos animais.

Até o final do século XIX, a calorimetria limitava-se a medir a produção de calor em animais (Webb, 1985) enquanto que os aparelhos (câmaras) respiratórios já tinham sido utilizados para experimentos em pessoas com diabetes, anemia e malária. No período entre 1892 e 1899, nos Estados Unidos, Atwater \& Rosa desenvolveram o primeiro calorímetro humano utilizando-se também do aparelho de Pettenkofer-Voit em conexão ao calorímetro (Boothby $\&$ Sandiford, 1920). Este aparelho era grande o suficiente para manter, confortavelmente, em seu interior um homem por duas semanas ou mais e era provido de cama, cadeira, mesa e um ciclo-ergômetro. As medições, então, poderiam ser feitas com o indivíduo em repouso, trabaIhando ou exercitando-se. Posteriormente, em 1905, Atwater \& Benedict aperfeiçoaram este calorímetro para que fosse possível a determinação simultânea também do consumo de oxigênio. A medida do oxigênio consumido, ou seja, a calorimetria indireta, significou importante melhora na mensuração da produção de caIor (Boothby \& Sandiford, 1920) pois possibilitava quantificar o oxigênio utilizado na oxidação dos substratos energéticos, além da possibilidade de se obter o consumo de energia nas várias atividades, ao in vés do gasto energético total de um intervalo de tempo grande.

Benedict, em 1907, assume a direção do Laboratório de Nutrição do Carnegie Institution of Washington, localizado em Boston, Estados
Unidos, dedicando-se à mensuração das trocas respiratórias. Ele construiu um cal orímetro respiratório e realizou alguns estudos com pacientes diabéticos, porém seu interesse se voltou para a medição da taxa metabólica basal em indivíduos saudáveis. Benedict continuou a aperfeiçoar as técnicas da calorimetria indireta, além de testar vários aparelhos respiratórios e desenvolver um próprio para uso em hospitais (Boothby \& Sandiford, 1920).

Em 1919, Harris \& Benedict publicaram os dados de taxa metabólica basal de 333 indivíduos, empregando pela primeira vez a análise estatística mais sofisticada disponível à época em dados fisiológicos. Desta análise foram deduzidas as primeiras equações de predição da taxa metabólica basal em homens, mulheres e crianças que serviriam de controle para comparação em situações especiais de dieta ou doença.

Nesta época, a TMB medida por calorimetria direta ou indireta servia como diagnóstico, principalmente, de distúrbios da tireóide. Com o passar do tempo, e com o advento de outros métodos para o diagnóstico de problemas da tireóide (Bursztein et al., 1989) o interesse pelo metabolismo basal diminuiu e a calorimetria direta passou a ser utilizada, principalmente, para estudos sobre a regulação da temperatura corporal, interesse que se estendeu até após a segunda guerra mundial. É a partir da década de 70, que uma nova era se inicia com os estudos de balanço energético e mais adiante, nos anos 80, a calorimetria indireta passa a ser utilizada na determinação dos requerimentos energéticos em pacientes graves submetidos à nutrição enteral e parenteral (Bursztein et al., 1989).

A retomada do interesse em medir a TMB de indivíduos saudáveis, surgiu após um grupo de expertos da FAO e Organização Mundial da Saúde (OMS) ter determinado que as necessidades energéticas deveriam ser calculadas com base no gasto energético diário, expresso como múltiplos da TMB (FAO/WHO/ UNU, 1985). Para tal finalidade foram desen volvidas equações de predição da TMB que foram recomendadas para uso internacional, fundamentalmente, pela ainda pouca disponibilidade de calorímetros indiretos para sua medição.

\section{Fund amentação teórica \\ da calorimetria indireta}

A calorimetria indireta é o método onde o gasto energético é estimado através da medição das trocas respiratórias, ou seja, do volume de oxigênio consumido $\left(\mathrm{V}_{2}\right)$ e do volume de gás 
carbônico produzido ( VंCO $_{2}$ ) (Ferrannini, 1988; Murgatroyd et al., 1993). Ela baseia-se nos princípios de que não existe uma reserva apreciável de oxigênio no organismo; que o oxigênio consumido reflete a oxidação dos nutrientes e que toda a energia química no organismo é proveniente da oxidação de carboidratos, gorduras e proteínas (Green, 1994). A quantidade de oxigênio utilizada para a oxidação ea produção de gás carbônico, dependerá do substrato que está sendo oxidado. O quociente respiratório $\left(\mathrm{QR}=\dot{\mathrm{V}} \mathrm{CO}_{2} / \mathrm{V}_{2}\right)$ varia entre 0,7 , durante a oxidação de gordura, e 1 quando apenas houver a oxidação de carboidratos. Para converter os valores de $\dot{\mathrm{VO}}_{2}$ e $\mathrm{V}_{\mathrm{CO}}$ em energia pode ser utilizada a tabela de Zuntz, elaborada no início do século XX (Lusk, 1917) que fornece os equival entes energéticos por litro de oxigênio consumido em relação a cada QR não protéico. Em situações em que só o $\mathrm{V}_{2}$ é medido, assume-se um equivalente energético de $5 \mathrm{kcal}$ por litro de oxigênio consumido. Quando tanto o $\dot{V}_{2}$ quanto o $\dot{V} \mathrm{CO}_{2}$ (ambos em $\mathrm{l} / \mathrm{min}$ ) são disponíveis, pode-se utilizar a equação proposta por Weir (1949): [(3,9 Xن்O $\left.\left.{ }_{2}\right)+\left(1,1 \times \dot{V} \mathrm{VO}_{2}\right)\right]$ que é um cálculo muito mais prático, dispensando a medição do metabolismo protéico ao incorporar um fator de correção pela sua não medição.

Fatores que influenciam a TM B ou sua medição

A TMB, por definição, deve ser medida controlando-se vários fatores, alguns óbvios, como a atividade física prévia, a ingestão alimentar ea temperatura e o nível de ruído ambiental; outros mais sutis, como o tabagismo e o período no ciclo menstrual. Quando a medição é realizada sem o controle destes fatores, costuma-se chamar o valor obtido de taxa metabólica de repouso. Entretanto, algumas características inerentes aos indi víduos sendo avaliados, como a idade, a aptidão física e a dimensão e composi ção corporais, podem explicar as diferenças interindividuais na TMB. A influência desses fatores na TMB serão revisados brevemente a seguir.

Dimensão e composição corporais

A TMB sofre influência das características individuais como a dimensão (massa corporal, estatura e superfície corporal - que é uma medida derivada da massa corporal e da estatura) e composição corporais dos indivíduos (Censi et al., 1998; FAO/WHO/UNU, 1985). A massa cor- poral apresenta uma correlação quase sempre elevada com a TMB, mas que varia bastante (coeficiente de correlação entre 0,45 a 0,96; Harris \& Benedict, 1919; Kendrick et al., 1990; Ravussin et al., 1982). A superfície corporal foi a primeira medida utilizada para expressar a TMB seguindo a Lei da Superfície Corporal, estabelecida por Rubner, em 1883, a partir de estudos em animais, que postulava que a produção de cal or era constante por unidade de superfície corporal (Du Bois, 1936; Harris \& Benedict, 1919). Desta forma, animais de tamanhos variados teriam a mesma produção de calor por unidade de área de superfície corporal. Harris \& Benedict (1919) questionaram a aplicabilidade desta lei, ao demonstrar que o metabolismo basal tinha uma correlação elevada tanto com a área de superfície corporal quanto com a massa corporal não sendo, porém, constante por unidade de superfície corporal como previamente estabelecido e nem independente da massa corporal como acreditava-se.

A expressão da TM B por unidade de massa e superfície corporais é uma prática muito comum, facilitando a comparação dos valores de TMB entre indivíduos com dimensões diferentes. Contudo, esta maneira de expressar a TMB não é perfeita pelo fato de não conseguir eliminar as diferenças interindividuais decorrentes de diferentes composições corporais (Censi et al., 1998; Cunningham, 1982). A massa corporal engl oba tecidos de diversas atividades metabólicas, como a gordura corporal, que apresenta um consumo de oxigênio desprezível, e a massa livre de gordura, componente da massa corporal responsável pelo maior consumo de oxigênio. A massa livre de gordura, por sua vez, é constituída por órgãos e tecidos que diferem quanto à atividade metabólica. A massa extracelular possui baixa atividade metabólica (Cunningham, 1982) e a massa celular corporal , também chamada de massa celular ativa, que em um indivíduo saudável corresponde entre 50 a $60 \%$ da massa livre de gordura, é responsável pela maior parte do metabolismo e compreende os componentes celulares das vísceras, cérebro, sangue e massa muscular (Ellis, 1996) que apresentam gastos en ergéticos distintos. As vísceras correspondem entre 20 e $30 \%$ da massa corporal de um adulto saudável (Shephard, 1991) sendo responsáveis por quase $60 \%$ da TMB. A massa muscular, que representa a maior parte da massa corporal, é responsável por apenas $20 \%$, no máximo $25 \%$, do consumo de oxigênio em condições basais(Brozek \& Grande, 1955; Durnin \& Passmore, 1967).

Em indivíduos obesos, parece queo aumento da TMB não é diretamente proporcional ao 
aumento da massa livre de gordura (Prentice et al., 1996). Uma explicação para tal fato é que na obesidade os órgãos metabolicamente ativos não se hipertrofiam ou aumentam em menor escala do que a massa muscular, alterando assim a composição da massa livre de gordura. Portanto, a TMB depende da quantidade e da atividade metabólica da massa celular corporal, assim como da sua proporção em relação à massa livre de gordura (Censi et al., 1998; FAO/ WHO/UNU, 1985; Kendrick et al., 1990). Apesar de existirem sugestões de que a TMB seja expressa em função da massa livre de gordura e não da massa corporal total (Piers et al., 1998) são ainda poucos os dados disponíveis de TMB e de composição corporal no mesmo grupo de indivíduos, devido, principalmente, à limitação metodológi ca da medição da composição corporal.

- Idade

A diminuição da TMB relacionada com a idade foi reconhecida já por Harris \& Benedict (1919), ao documentarem uma correlação negativa entre TMB e idade para homens $(r=-30)$ e muIheres ( $r=-20)$ fato que fez os autores incluírem a idade na equação de predição para corrigir os valores estimados.

O declínio da TMB com a idade parece ser um reflexo das alterações na composição corporal que ocorrem com o envelhecimento, tais como a diminuição da massa muscular e o aumento da massa de gordura, sem, aparentemente, modificação na massa visceral. Tzankoff \& Norris (1977) evidenciaram um decréscimo do consumo de oxigênio que foi proporcional à diminuição da massa muscular em homens para cada década após os 45 anos de idade. Este achado foi, posteriormente, confirmado por um estudo longitudinal no qual foram acompanhados 355 homens por 26 anos (Tzankoff \& Norris, 1978). Mais recentemente, Piers et al. (1998) questionam se a redução na TMB com o envel hecimento seja causada, exclusivamente, pela redução da quantidade de massa livre de gordura ou se ocorrem alterações qualitativas do tecido, visto que, aTMB expressa em função da massa livre de gordura ainda é menor em idosos comparativamente a adultos jovens.

\section{- Atividade física}

Durante a atividade física há aumento do consumo de oxigênio que pode manter-se por várias horas após o término do exercício, elevando o gasto energético no repouso. Este fenôme- no é conhecido como débito de oxigênio (ou consumo de oxigênio excessivo pós exercício) e sua duração dependerá da intensidade e duração do exercício realizado (Sedlock et al., 1989). Entretanto, o aumento da TMB após uma sessão de exercício parece ser transitório e não se manteria por mais de 24 horas (Bingham et al., 1989; Wilmore et al., 1998). Broeder et al. (1992) observaram valores de TMB significativamente maiores, medidos após 14 horas de um treinamento físico, em relação aos valores obtidos na fase de pré-treinamento, não sendo diferentes, entretanto, quando a taxa foi medida 48 horas após o exercício. Weststrate et al. (1990) também não evidenciaram um efeito prolongado e sistemático na TMB em indivíduos submetidos a uma sessão de exercício de intensidade moderada, e outro estudo apenas verificou um pequeno aumento (3,9\%) na TMB em indivíduos submetidos a uma sessão de exercício intenso (Goldberg et al., 1990).

Apesar dos estudos não serem conclusivos quanto à relação entre o condicionamento físico eTMB, especula-se que a prática de atividade física regular possa aumentar a TMB em conseqüência da adaptação crônica ao exercício, visto que foi encontrada uma correlação positiva entre a TMB e o consumo máximo de oxigênio ( $\dot{V}_{2}$ máximo) (Burke et al.,1993). O aumento na TMB, no entanto, parece ocorrer apenas em atletas altamente treinados, i.e., $\mathrm{V}_{2}$ máximo $\geq 70 \mathrm{ml}^{.} \mathrm{kg}^{-1}$.min-1 (Poehlman et al., 1988a) já que em indivíduos não atletas submetidos a treinamento físico com duração variando entre 9 e 20 semanas, não foram evidenciadas al terações significativas na TMB quando expressa em relação à massa livre de gordura (Bingham et al., 1989; Broeder et al., 1992; Wilmore et al., 1998).

É importante, então, quando se pretende medir a TMB, que os indivíduos sejam orientados a manter as suas atividades cotidianas e a evitar qualquer tipo de atividade física intensa durante o dia que precede o teste, com o intuito de anular qualquer efeito agudo do exercício na TMB. Durante a medição da taxa, o indivíduo sendo submetido à medição deve ser orientado a permanecer relaxado, assi m como, informado dos detalhes do procedimento para evitar a tensão proveniente do desconhecimento do mesmo (Boothby $\&$ Sandiford, 1920).

\section{- Dieta}

O aumento do consumo de oxigênio após a ingestão de alimentos já havia sido descrito por Lavoisier em seus estudos sobre o metabolismo no homem (King, 1924). Este aumento do 
gasto energético é devido ao processo de digestão, absorção e armazenamento dos nutrientes ingeridos, principalmente a proteína, e recebe o nome de termogênese induzida pela dieta ou efeito térmico dos alimentos, ou ainda, ação dinâmica específica dos alimentos (FAO/WHO/ UNU, 1985).

Relatos do começo do século XX (Boothby $\&$ Sandiford, 1920) indicavam um aumento de $46 \%$ na produção de calor após ingestão de uma refeição rica em proteínas, efeito que permanecia por cerca de 12 horas. Isto poderia sugerir que a TMB, em indivíduos vegetarianos, fosse menor pela pequena ingestão protéica, hipótese que foi utilizada para explicar as diferenças de TMB encontradas entre indianos e europeus e norte-americanos (Soares \& Shetty, 1988). Entretanto, Harris \& Benedict (1919) não observaram diferenças significativas de TMB entre vegetarianos e não vegetarianos e, mais recentemente, Poehlman et al. (1988b) evidenciaram o mesmo, o que parece indicar que o efeito no metabolismo se restringe a pouco tempo após a ingestão protéica.

Com o objetivo de avaliar a influência tardia da ingestão protéica no metabolismo basal, Soares et al. (1988) conduziram um estudo em homens que receberam dietas iso-energéticas por três dias, porém com quantidades diferenciadas de proteína $(0,9 ; 1,2$ e 1,5g/ kg/ dia) para cada dia, com a TMB sendo medida no dia seguinte, após 12 horas de jejum. Os autores não observaram nenhum efeito da dieta sobre a TMB. Estas evidências sugerem que não há necessidade de padronização da dieta no dia que precede a medição da TMB, porém o jejum é fundamental pois a presença do alimento no trato gastrointestinal eleva o gasto energético pela ação dinâmica dos al imentos. Um intervalo de 12 horas parece ser suficiente para que o processo de digestão esteja finalizado (Bursztein et al., 1989) e, desta forma, não afete aTMB.

\section{- Clima}

O clima foi durante muito tempo, utilizado para explicar as diferenças encontradas entre a TMB de populações residentes em diferentes regiões do mundo, baseado, fundamentalmente, em estudos real izados na primeira metade do século XX. Almeida (1919, 1920) publicou os dados de TMB medidos em vinte homens brasileiros, residentes no Rio de Janeiro, evidenciando que os valores encontrados foram em média $20 \%$ menores do que o valor de homens norte-americanos. A partir destes estudos, o autor concluiu que o metabolismo basal de indivíduos nascidos e residentes em regiões tro- picais era menor do que o de indivíduos de regiões de climas frio e temperado. Esta constatação foi reforçada, segundo Almeida (1924), pelos achados de Montoro que encontrou valores de TM B 15,8 e 12,9\% menores do que os estimados pela equação de Harris \& Benedict (1919) para homens e mulheres cubanos.

Outros estudos apoiaram a idéia de que o metabolismo basal de indivíduos das regiões tropicais era men or do que em climas temperados. Galvão (1948) mediu a TMB em cinqüenta homens resi dentes em São Paulo (cidade localizada sobre o Trópico de Capricórnio) e constatou valores menores quando comparados a de homens norte-americanos de massa corporal semelhante. As diferenças variaram entre 5 a 12\% havendo uma tendência a diminuir com o aumento da massa corporal. Mais recentemente, uma análise de dados de TMB em indivíduos residentes em países tropicais obtidos da literatura (Henry \& Rees, 1991) demonstrou que os valores medidos eram em mé dia $8 \%$ menores do que os estimados pela equação de Schofield (1985) que foi derivada de uma amostra composta, principalmente, por europeus e norte-americanos.

Em contraposição a estes achados, crescem as evidências de que não existem diferenças entre a TMB em indivíduos vivendo em diferentes regiões climáticas. Lawrence et al. (1988) não evidenciaram diferenças significativas entre a TMB medida em mulheres da Escócia, Gâmbia e Tai lândia que apresentavam valores de massa livre de gordura semelhantes. Da mesma forma, não foram encontradas diferenças entre a TMB de indianos e norte-americanos, quando as comparações foram realizadas com base em dados pareados para idade e massa corporal (Soares et al., 1993). Em um estudo longitudinal (até seis meses) comparativo entre recém-imigrados à I nglaterra e nativos, Hayter \& Henry (1993) não encontraram diferença significativa entre os grupos na taxa expressa em relação à massa corporal .

Talvez a interpretação quanto a diferenças da TMB entre indivíduos residentes em países tropicais e temperados tenha sido equivocada por ser baseada, principalmente, na comparação entre valores medidos e estimados por padrões ou equações derivadas de europeus e norte-americanos e não, necessariamente, entre indivíduos com características semelhantes de idade, massa corporal e composição corporal. Além disso, há evidências de que as equações de Harris \& Benedict (1919) assim como a de Schofield (1985) também superestimam a TMB em norte-americanos e europeus (Clark \& Hoffer, 1991; de Boer et al., 1988) indicando 
desta forma, possíveis problemas relacionados às próprias equações, fazendo com que estas não fossem adequadas para apontar diferenças entre indivíduos submetidos a diferentes climas. Desta maneira, parece que a melhor forma para se verificar possíveis diferenças de TMB em função do clima seja comparar os valores obtidos com um grupo controle pareado para idade, gênero, massa corporal e composição corporal e, se possível, que a TMB tenha sido medida utilizando-se a mesma metodologia.

Recentemente, Wahrlich \& Anjos (2000) encontraram valores de TMB em 45 mulheres jovens de Porto Alegre, Rio Grande do Sul (30॰ 01'59" Sul e 51013'48" Oeste) iguais aos descritos para mulheres da mesma idade vivendo no Rio de Janeiro (22053' Sul e 43006'13" Oeste) descritos por Cruz et al . (1999). Cabe ressaltar que ambos os estudos utilizaram os mesmos equipamentos, seguindo o mesmo protocolo. Assim, parece que as diferenças climáticas, regionais e, talvez, étnicas não parecem influenciar o metabolismo basal.

\section{- Tabagismo}

Geralmente, o grupo de indivíduos fumantes apresenta uma massa corporal média menor quando comparada a de não fumantes (Wack \& Rodin, 1982). A menor massa corporal poderia ser explicada por alterações no gasto energético que seriam induzidas por um aumento da TMB, em função do tabagismo ou pelo próprio efeito térmico do fumo (Dalloso \&James, 1984).

A nicotina contida no cigarro parece elevar a TMB, visto que a inalação isolada desta substância por fumantes, resulta em aumento de $6 \%$ nos valores medi dos (Perkins et al., 1989). Em outro estudo, o consumo de um cigarro causou um aumento de 3\% da TMB em relação ao grupo controle, aumento que permaneceu por trinta minutos (Dalloso \& James, 1984). Foi evidenciado que o efeito dos cigarros consumidos ao longo do dia persiste durante a noite (Warwick \& Busby, 1993) não afetando, entretanto, a TMB medida na manhã seguinte. Mais recentemente, Warwick et al. (1995) não encontraram evidência para um efeito crônico do fumo em qualquer dos componentes do gasto energético diário em indivíduos fumantes, em períodos de fumo e de não fumo (4 a 8 semanas) avaliados num calorímetro respiratório.

Desta forma, parece ainda existir controvérsias sobre a inter-relação entre o tabagismo e o aumento do gasto energético. Entretanto, apesar de existir um efeito agudo da nicotina e do fumo sobre a TMB, a abstinência de 12 horas pode ser suficiente para anular o aumento agudo do gasto energético decorrente do consumo de cigarros.

- Ciclo menstrual

A investigação sobre a existência de uma periodicidade daTMB em função do ciclo menstrual é de interesse científico desde o início do século XX. Snell et al . (1920) referiram um aumento médio de $10 \%$ naTM $B$ em dez mulheres no período pré-menstrual e durante a menstruação, seguido de um declínio no período pós-menstrual. Porém, vários estudos conduzidos posteriormente não evidenciaram nenhuma alteração significativa da TMB durante o ciclo menstrual (Wiltshire, 1921) a não ser um pequeno declínio dos valores durante a menstruação (Benedict \& Finn, 1928; Blunt \& Dye, 1921; Hitchcock \& Wardwell, 1929). Apesar das conclusões divergentes, os dados de estudos conduzidos durante a década de 20 , concordavam no aspecto que se refere à observação de um aumento da TMB no período pré-menstrual que poderia estar vinculado à ovulação (Hitchcock \& Wardwell, 1929).

Mais recentemente, Solomon et al . (1982) mediram a TMB num grupo de seis mulheres durante as fases folicular e luteal que foram determinadas a partir da duração do ciclo menstrual de cada mulher. A TMB em cinco delas variou significativamente durante o ciclo menstrual, sendo observado um aumento dos valores na fase luteal do ciclo, confirmando assim, os achados prévios que indicavam um aumento no período pré-menstrual. Bisdee et al. (1989) também observaram um aumento brusco da TMB entre a fase folicular tardia e a fase luteal inicial. O aumento daTMB poderia ser atribuído, em parte, a alterações dos níveis de progesterona que eleva a produção de calor corporal, já que a secreção deste hormônio coinci de com as alterações observadas na TMB (Solomon et al., 1982). Entretanto, outros estudos não evidenciaram diferenças significativas entre as fases do ciclo menstrual (Piers et al., 1995; Tai et al., 1997).

Outro aspecto que deve ser considerado é o possível efeito na TMB de mulheres usuárias de anticoncepcional oral. Curtis et al. (1996) observaram valores de TMB mais estáveis durante todo o ciclo menstrual em mulheres que usaram anticoncepcional oral. Entretanto, Diffey et al. (1997) mostraram que mulheres usuárias de anticoncepcional oral tinham a TMB maior em, aproximadamente, $5 \%$ do que muIheres não usuárias do mesmo medicamento com as mesmas características físicas e de composição corporal. Desta forma, nas mulheres 
em idade fértil não usuárias de anticoncepcional oral, parece haver uma maior variabilidade daTMB resultante da influência do ciclo menstrual. Contudo, esta variação pode ser muito pequena em relação às flutuações diárias causadas por outros fatores (Solomon et al., 1982). As discrepâncias entre estudos fazem com que seja recomendável a padronização quanto ao período do ciclo menstrual em que serão realizadas as medições de TMB. Este procedimento permite a comparação da TMB entre as mulheres, evitando possíveis diferenças decorrentes do efeito do ciclo menstrual sobre os valores medidos.

\section{- Ambiente do teste}

As medições de TMB devem ser real izadas após uma noite de sono (Bursztein et al., 1989) e, de preferência, logo após o indivíduo acordar. Para se obter a medição imediatamente apósacordar seria necessário que o indivíduo dormisse no laboratório ou então, o pesquisador se deslocasse até a residência do indivíduo. Ambas as situações, entretanto, são artificiais e podem interferir nos resultados. O fato de dormir num ambiente e cama diferentes pode afetar a qualidade do sono alterando os valores de TMB (Turley et al., 1993) e o mesmo poderia ocorrer pela expectativa do indivíduo em receber o pesquisador em casa. Alternativamente, e o que é realizado na maioria dos estudos, os indivíduos dormem em suas residências e são instruídos a se deslocarem até o laboratório com o mínimo de esforço. Apesar de Berke et al. (1992) terem observado um aumento de $8 \%$ na TMB dos indivíduos que passaram a noite em casa e se deslocaram até o laboratório, outros estudos não evidenciaram diferenças significativas entre os valores obti dos quando os indivíduos passaram a noite em casa e se deslocaram até o local em que seria realizada a medição, do que quando dormiram no próprio laboratório (Bandini et al., 1995; Figueroa-Colon et al., 1996; Turley et al., 1993).

Contudo, a medida da TMB só deve ser iniciada após um período de repouso para diminuir qualquer efeito da atividade física recente, como o ato de vestir-se, dirigir ou caminhar. Harris \& Benedict (1919) recomendavam um período mínimo de trinta minutos. Porém, Boothby \& Sandiford (1920) observaram que não havia diferença entre os valores da TMB pelo repouso preliminar superior a vinte minutos e concluíram que este período seria adequado para atingir o nível basal se nenhum exercício físico extremo precedesse o teste. $\mathrm{Na}$ prática, um tempo maior do que este pode fa- zer com que o indivíduo durma ou fique impaciente.

A temperatura do ambiente também é importante na medição de TMB, visto que a temperatura acima ou abaixo da zona térmica de neutralidade, citada por Henry \& Emery (1986) como sendo de 25 a $26 \circ C$, aumenta o gasto energético através do suor ou tremor para manter a temperatura corporal constante (Bursztein et al., 1989). Um estudo em mulheres submetidas à calorimetria direta durante trinta horas aval iou os efeitos da temperatura no gasto energético eTMB em duas ocasiões com as mulheres vestidas com uma roupa fina de algodão (Dauncey, 1981). Foi evidenciado um aumento de cerca de $11 \%$ nos valores de TMB quando as mulheres permaneceram no calorímetro direto com temperatura de $22 \circ \mathrm{C}$ (temperatura suficiente para evitar o tremor) em relação aos valores obtidos nas mesmas mulheres quando expostas a uma temperatura de $280 \mathrm{C}$.

Em ambientes em que a temperatura está abaixo da zona de neutralidade o uso de roupa adequada pode ser suficiente para evitar o aumento do gasto energético devido ao efeito térmico do frio que produz o tremor e o eriçamento dos pêlos corporais.

Equações de predição:

descrição e validade

Na literatura são encontradas diversas equações de predição da TMB. NaTabela 1 são apresentadas as equações de Harris \& Benedict (1919), Schofield (1985), FAO/WHO/UNU (1985) e Henry \& Rees (1991), o número de indivíduos cujos dados foram usados na derivação das respectivas equações, assim como o coeficiente de determinação (R2) para cada uma delas. Observando as equações, é digno de nota que, geralmente: (1) existem mais dados para homens do que para mulheres; (2) os R2 são maiores para os homens; e (3) os R2 são baixos, principalmente para as equações nos indivíduos após os 18 anos de vida.

As primeiras equações foram publicadas em 1919 por Harris \& Benedict, derivadas de uma amostra composta por 136 homens, 103 mulheres e 94 crianças de origem norte-americana, considerados saudáveis, e obtidos através de calorimetria indireta. Os dados utilizados nas análises foram coletados no Laboratório de Nutrição do Carnegie Institution of Washington e em outros laboratórios associados, todos realizados com técnicas padronizadas e em aparelhos semelhantes. As medidas foram feitas com os indivíduos em jejum de 12 horas, 
Tabela 1

Equações de predição da taxa metabólica basal, com o número amostral (n) e o coeficiente de determinação (R2), propostas por Harris \& Benedict (1919), Schofield (1985), FAO/WHO/UNU (1985) e Henry \& Rees (1991).

\begin{tabular}{|c|c|c|c|c|c|}
\hline & Faixa Etária (anos) & Gênero & Equação & $\mathrm{n}$ & $\mathrm{R}^{2}$ \\
\hline \multirow[t]{3}{*}{ Harris \& Benedict (1919) } & & & (kcal/dia) & & \\
\hline & 15 a 74 & Masculino & $66,4730+13,7516(M C)+5,0033(E)-6,7550(I)$ & 136 & $0,7534 *$ \\
\hline & 15 a 74 & Feminino & $655,0955+9,5634(M C)+1,8496(E)-4,6756(I)$ & 103 & $0,5337 *$ \\
\hline \multirow[t]{13}{*}{ Schofield (1985) } & & & $(M J / d i a)^{1}$ & & \\
\hline & $<3$ & Masculino & $0,249(M C)-0,127$ & 162 & $0,9025^{* * *}$ \\
\hline & & Feminino & $0,244(M C)-0,130$ & 137 & 0,9216 \\
\hline & $3 \vdash 10$ & Masculino & $0,095(M C)+2,110$ & 338 & 0,6889 \\
\hline & & Feminino & $0,085(M C)+2,033$ & 413 & 0,6561 \\
\hline & $10 \vdash 18$ & Masculino & $0,074(M C)+2,754$ & 734 & 0,8649 \\
\hline & & Feminino & $0,056(M C)+2,898$ & 575 & 0,6400 \\
\hline & $18 \vdash 30$ & Masculino & $0,063(M C)+2,896$ & 2.879 & 0,4225 \\
\hline & & Feminino & $0,062(M C)+2,036$ & 829 & 0,5329 \\
\hline & $30 \vdash 60$ & Masculino & $0,048(M C)+3,653$ & 646 & 0,3600 \\
\hline & & Feminino & $0,034(M C)+3,538$ & 372 & 0,4624 \\
\hline & $\geq 60$ & Masculino & $0,049(M C)+2,459$ & 50 & 0,5041 \\
\hline & & Feminino & $0,038(M C)+2,755$ & 38 & 0,4624 \\
\hline \multirow[t]{13}{*}{ FAO/WHO/UNU (1985)** } & & & $(M) / d i a)^{1}$ & & \\
\hline & $<3$ & Masculino & $0,255(M C)-0,226$ & & $0,9409 * * *$ \\
\hline & & Feminino & $0,255(M C)-0,214$ & & 0,9409 \\
\hline & $3 \vdash 10$ & Masculino & $0,0949(M C)+2,07$ & & 0,7396 \\
\hline & & Feminino & $0,0941(M C)+2,09$ & & 0,7225 \\
\hline & $10 \vdash 18$ & Masculino & $0,0732(M C)+2,72$ & & 0,8100 \\
\hline & & Feminino & $0,0510(M C)+3,12$ & & 0,5625 \\
\hline & $18 \vdash 30$ & Masculino & $0,0640(M C)+2,84$ & & 0,4225 \\
\hline & & Feminino & $0,0615(M C)+2,08$ & & 0,5184 \\
\hline & $30 \vdash 60$ & Masculino & $0,0485(M C)+3,67$ & & 0,3600 \\
\hline & & Feminino & $0,0364(M C)+3,47$ & & 0,4900 \\
\hline & $\geq 60$ & Masculino & $0,0565(M C)+2,04$ & & 0,6241 \\
\hline & & Feminino & $0,0439(M C)+2,49$ & & 0,5476 \\
\hline \multirow[t]{9}{*}{ Henry \& Rees (1991) } & & & $(\mathrm{MJ} / \mathrm{dia})^{1}$ & & \\
\hline & $3 \vdash 10$ & Masculino & $0,113(\mathrm{MC})+1,689$ & 196 & $0,5625^{* * *}$ \\
\hline & & Feminino & $0,063(M C)+2,466$ & 88 & 0,1681 \\
\hline & $10 \vdash 18$ & Masculino & $0,084(M C)+2,122$ & 409 & 0,6400 \\
\hline & & Feminino & $0,047(M C)+2,951$ & 233 & 0,3969 \\
\hline & $18 \vdash 30$ & Masculino & $0,056(M C)+2,800$ & 1.174 & 0,3481 \\
\hline & & Feminino & $0,048(M C)+2,562$ & 350 & 0,4489 \\
\hline & $30 \vdash 60$ & Masculino & $0,046(M C)+3,160$ & 274 & 0,4356 \\
\hline & & Feminino & $0,048(M C)+2,448$ & 98 & 0,5929 \\
\hline
\end{tabular}

$\mathrm{MC}=$ massa corporal $(\mathrm{kg}) ; \mathrm{E}=$ estatura $(\mathrm{cm}) ; \mathrm{I}=$ idade (anos).

* calculado pelos autores.

** n não disponível.

*** calculado pelos autores a partir do R publicado.

1 Para converter em kcal, multiplicar o resultado por 239 
em posição supina e em repouso muscular. Cada indivíduo era submetido a várias medições com duração de 15 minutos cada e com um intervalo de vinte minutos entre elas. O menor valor de TMB era selecionado para análise por acreditar-se que este representaria o metabolismo mais próximo do basal para o indivíduo. As equações desenvolvidas, uma para cada sexo, incluíram como variáveis independentes a massa corporal, a estatura e a idade.

Até hoje as equações de Harris \& Benedict são utilizadas, principalmente, na prática clínica (Bursztein et al., 1989) para determinar os requerimentos energéticos de indivíduos com as mais diversas doenças. Entretanto, há vários estudos que demonstram que estas equações são inadequadas para estimar a TMB. O próprio Benedict, menos de dez anos após a publicação de suas pesquisas, já havia evidenciado que a equação de predição em mulheres geralmente fornecia uma estimativa superior ao valor medido e sugeriu que os val ores estimados pela equação fossem reduzidos em $5 \%$ (Benedict, 1928). Mais recentemente, foi verificado que em um grupo de mulheres americanas a equação fornecia estimativas 14\% superiores à TM B medida (Owen et al., 1986) enquanto que em homens a superestimativa ficou em torno de 9\% (Clark \& Hoffer, 1991; Owen et al., 1987).

Em 1985, Schofield compilou os dados de TMB disponíveis até então, com o objetivo de derivar equações de predição adequadas para uso internacional e que seriam recomendadas pelo Comitê da FAO/OMS para estimar as necessidade energéticas. Assim, Schofield (1985) juntou os dados de 114 estudos que foram considerados cientificamente adequados e sobre os quais existiam um mínimo de dados relevantes (idade, gênero, estatura e massa corporal). Apesar do rigor na seleção dos dados, o autor incluiu análises anteriormente realizadas em dados compilados, baseados em uma única avaliação e outros que eram a média de duas ou mais medidas, além de alguns poucos $(1,4 \%)$ que eram o menor valor de TMB e outros sobre os quais não se tinha informações sobre o número de avaliações. Através deste procedimento foi obtida uma amostra final de 7.173 indivíduos (4.809 homens e 2.364 mulheres) em sua mai oria de origem européia ou norte-americana (Henry \& Rees, 1991). Schofield (1985) testou modelos de estimativa de TMB desenvolvidos em estudos anteriores e explorou a relativa contribuição potencial de diferentes variáveis independentes (estatura e massa corporal) e medidas complexas construídas a partir destas (área de superfície corporal) além das informações de idade e gênero. Essas variáveis foram escolhidas por serem mais simples de se obter e por terem significado fisiológico e prático em termos de quantidade de variação de TMB estimada, além de gerarem equações com erros de estimativa menores. Na computação das equações finais o autor decidiu pelo uso de faixas etárias utilizadas previamente em outros estudos ( 0 a 3; 3 a 10; 10 a 18; 18 a 30; 30 a 60 e >60 anos) que representassem períodos com significado clínico na vida humana (Tabela 1).

Schofield (1985) propôs um modelo de equação que util izava somente a massa corporal, visto que a introdução de outras variáveis (estatura, área de superfície corporal) pouco melhoravam a estimativa da TMB, i.e., não aumentavam substancialmente o valor de $\mathrm{R}^{2}$. Estas equações foram, posteriormente, adotadas pela OMS com uma pequena modificação, já que o banco de dados original de Schofield (1985) foi ampliado para 11.000 dados de TMB (FAO/WHO/UNU, 1985). Entretanto, há evidências de que as equações propostas pela OMS não sejam adequadas para estimar a TMB em indivíduos de diferentes partes do mundo (Cruz et al., 1999; Henry \& Rees, 1991; McNeill et al., 1987; Piers \& Shetty, 1993; Piers et al., 1997; Valencia et al., 1994; Wahrlich, 2000). Schofield (1985) em seu trabalho já apontava para esta possível inadequação, visto que na tentativa de validar suas equações, ele próprio verificou que quando elas foram aplicadas a uma amostra de indianos houve uma superestimativa da TMB em torno de $11,2 \%$ para os homens e 9,9\% para as mulheres. Nesta análise, Schofield (1985) ressaltou que a TMB dos indivíduos de países em desenvolvimento (Índia e China) era menor em termos absolutos, assim como, por unidade de massa corporal quando comparada a de indivíduos europeus e norte-americanos.

Há evidências de que as equações da FAO/ WHO/UNU (1985) superestimam, também, a TMB em pessoas que vivem em zonas temperadas (Clark e Hoffer, 1991; de Boer et al., 1988; Piers \& Shetty, 1993; Soares et al ., 1993). Uma análise detalhada do banco de dados utilizado por Schofield (1985) revela que a maioria dos indivíduos era ital ianos ( $50 \%$ da amostra) que apresentavam TMB maior do que os demais grupos, fato que pode ter introduzido viéses nas equações (Hayter \& Henry, 1993). Além disso, pode ser que tenha se incorporado medidas realizadas sem considerar as condições básicas de jejum e repouso, resultando em valores mais elevados de TMB (Valencia et al., 1994).

As evidências de que a TMB era superestimada pela equação de Schofield (1985) principalmente em regiões tropicais, fizeram com 
que Henry \& Rees (1991) derivassem equações de predição para indivíduos resi dentes nos trópicos. Os dados foram obtidos a partir de estudos da TMB encontrados na literatura que preenchessem os seguintes critérios: a TMB deveria ter sido medida sob condições padrão de repouso e jejum em indivíduos saudáveis; o equipamento utilizado deveria ter sido descrito; e os dados de idade, gênero, massa corporal e estatura dos indivíduos tinham que estar disponíveis. Ao final da seleção, foram reunidos 2.822 dados de indivíduos de ambos os sexos, de diversas faixas etárias, provenientes de regiões tropicais como China, Japão, Malásia, Havaí, América do Sul e outros. Foram derivadas, então, equações de regressão para 6 faixas etárias de cada sexo baseadas apenas na massa corporal. Apesar destas equações fornecerem estimativas menores do que as obtidas pelas de Schofield (1985) e da OMS (FAO/WHO/UNU, 1985) os valores por el as estimados parecem, ainda, superestimar a TMB em regiões tropicais como evidenciado em um grupo de sessenta indianas (4\%; Piers \& Shetty, 1993) e em cinqüenta universitárias do Rio de Janeiro (7\%; Cruz et al., 1999).

Uma análise de 16 estudos encontrados na literatura sobre a validação das equações de Schofiled (1985) ou da FAO/WHO/UNU (1985) através da calorimetria indireta, indica que em sua maioria (dez estudos, Tabela 2) a TMB era superestimada pelas equações. Essa característica ocorreu tanto para grupos de indivíduos residentes em regiões tropicais quanto temperadas como América do Norte e Europa, sendo que para essas populações os estudos de validação são mais escassos. Os valores percentuais de superestimativa observados variaram entre 2,2 e 13,5\%, sendo que em estudos que avaliaram ambos os sexos, as mulheres ten deram a ter valores percentuais de superestimativa menores quan do comparados aos dos homens de mesma faixa etária. É importante frisar que esses são valores médios de superestimativa e, que, mesmo nestes estudos, as equações podem ser adequadas ou subestimarem a TMB em alguns indivíduos. Por exemplo, Anjos et al. (1998) encontraram superestimativa de TMB (média de 14\%) em $80 \%$ das cinqüenta mulheres jovens do Rio de Janeiro avaliadas e subestimativa (média de 7,4\%) nas outras dez mulheres. Da mesma forma, Wahrlich (2000) concluiu que a TMB foi superestimada em $78 \%$ das sessenta mulheres resi dentes em Porto Alegre avaliadas e subestimativa nas restantes.

Os resultados dos outros seis estudos ( Tabela 3) conduzidos em quase sua totalidade em mulheres, indicaram adequação e, até mesmo, subestimativa da TMB pelas referidas equações. Foi observado que em mulheres africanas a equação da FAO/WHO/UNU (1985) subestimou a TMB entre 4 a 10\%. Uma explicação plausível para tal fato seria decorrente de uma composição corporal distinta, pois as mulheres africanas, em comparação com as norte-americanas e européias, teriam uma menor proporção de tecido adiposo em relação à massa livre de gordura (Lawrence et al., 1988).

Por outro lado, dois estudos conduzidos na Colômbia e Índia são contraditórios aos achados para as populações vivendo nos trópicos. Spurr et al. (1994) evidenciaram que a equação de Schofield foi adequada para estimar a TMB em 21 mulheres colombianas. Os autores especulam que tal resultado poderia ser explicado pelo fato destas mulheres residirem em uma altitude moderada que anularia o efeito de viverem nos trópicos. O estudo conduzido em homens e mulheres indianos afirma que as equações FAO/WHO/UNU são apropriadas para estimar a TMB (Ferro-Luzzi et al., 1997) e sugere que as diferenças entre raça, clima, dieta ou estado nutricional parecem não modificar a TMB não invalidando, desta forma, o poder de predição das equações da FAO/WHO/UNU. Apesar da posição taxativa neste úl timo estudo quanto às equações de predição da FAO/WHO/ UNU, ainda são maiores as evidências de que elas não seriam adequadas para uso internacional.

\section{Conclusão}

A TMB é fundamental para a determinação da necessidade energética de indivíduos e populações, além disso é aplicada em estudos epi demiológicos para validar as informações de ingestão alimentar e para se expressar o nível de atividade física. Geralmente, a TMB é estimada por equações de predição já que não existe grande disponibilidade de calorímetros indiretos. A maioria dos estudos de validação das equações de predição mostram uma inadequação das mesmas para a determinação da TMB em diferentes populações, apesar da tendência atual de se supor que não haja diferenças entre a TMB de diversas populações no mundo. As equações da FAO/WHO/UNU, indicadas para uso internacional, ten dem a superestimar a TMB, principalmente em populações de regiões tropicais, fato que tem sido explicado pelo reconhecimento da inadequação do banco de dados usado na geração das equações, tornando sua utilização universal questionável. Sendo assim, permanece a necessida- 
Estudos que demonstram que as equações para predição da Taxa Metabólica Basal sugeridas por Schofield (S)

e FAO/WHO/UNU, (F) superestimam os valores de TMB em populações residentes em regiões tropicais e temperadas.

\begin{tabular}{|c|c|c|c|c|c|}
\hline Referência & $\mathrm{n}$ & Sexo & Idade (anos) & $\begin{array}{l}\text { Superestimativa da TMB pelas equações } \\
\text { Schofield (S) ou FAO/WHO/UNU (F) }\end{array}$ & Origem \\
\hline McN eill et al. (1987) & 58 & M & $\chi=31$ & $12,1 \%(\mathrm{~F}) \mathrm{a}$ & Índia \\
\hline Soares \& Shetty (1988) & 123 & M & $18-30$ & $9,3 \%(S)^{b}$ & Índia \\
\hline Clark \& Hoffer (1991) & 29 & M & $18-33$ & $8,2 \%(S) c$ & Canadá \\
\hline \multirow[t]{8}{*}{ de Boer et al. (1988) } & 7 & M & $23-34$ & $9,8 \%(F)^{b}$ & Europa* \\
\hline & & & & $8,8 \%(\mathrm{~S})^{b}$ & \\
\hline & 8 & M & $27-40$ & $11,1 \%(F)^{b}$ & África* \\
\hline & & & & $9,8 \%(S)^{b}$ & \\
\hline & 7 & M & $28-44$ & $8,4 \%(F)^{b}$ & Ásia (M)* \\
\hline & & & & $6,9 \%(S)^{b}$ & \\
\hline & 8 & M & $18-31$ & $8,8 \%(F)^{b}$ & Ásia $(C)^{*}$ \\
\hline & & & & $8,4 \%(S)^{b}$ & \\
\hline \multirow[t]{4}{*}{ Henry \& Rees, (1991) } & 1.174 & M & $18-30$ & $10,3 \%(\mathrm{~F}) \mathrm{a}$ & Trópicos \\
\hline & 274 & M & $30-60$ & $11,2 \%(\mathrm{~F}) \mathrm{a}$ & \\
\hline & 350 & $\mathrm{~F}$ & $18-30$ & $3,8 \%(F) a$ & \\
\hline & 98 & $\mathrm{~F}$ & $30-60$ & $9,7 \%(F) a$ & \\
\hline \multirow[t]{2}{*}{ Piers \& Shetty (1993) } & 60 & $\mathrm{~F}$ & $18-30$ & $9,2 \%(S)^{b}$ & Índia \\
\hline & 52 & $\mathrm{~F}$ & $18-30$ & $4,1 \%(S)^{b}$ & $\begin{array}{l}\text { América } \\
\text { do Norte/Europa }\end{array}$ \\
\hline Valencia et al. (1994) & 32 & M & $18-40$ & $8,2 \%(F) d$ & México \\
\hline \multirow[t]{2}{*}{ Piers et al. (1997) } & 39 & M & $18-30$ & $5,6 \%(S) d$ & Austrália \\
\hline & 89 & $\mathrm{~F}$ & $18-30$ & $2,2 \%(S) d$ & \\
\hline Cruz et al. (1999) & 50 & $\mathrm{~F}$ & $19-27$ & $12,5 \%(F)^{d}$ & Brasil \\
\hline \multirow[t]{2}{*}{ Wahrlich (2000) } & 60 & $\mathrm{~F}$ & $20-40$ & $12,9 \%(S)^{d}$ & Brasil \\
\hline & & & & $13,5 \%(F)^{d}$ & \\
\hline
\end{tabular}

* TMB medida na Holanda, com dados de europeus da Holanda, da França e Asiáticos (M) = de origem Mongol

(China, Samoa O este, Indonésia) Vietnã e $(C)=$ de origem caucasiana (Sri Lanka e Bangladesh).

Cálculo do \% da superestimativa da TMB: a não é mencionado; b (TMB estimada - TMB medida/TMB estimada) x 100;

c 100 - [(TMB medida/TMB estimada) x 100]; d (TMB estimada - TMB medida/TMB medida) x 100.

Tabela 3

Estudos onde houve adequação ou subestimativa do valor da Taxa M etabólica Basal pelas equações de predição, sugeridas pela FAO/WHO/UNU em diversas populações.

\begin{tabular}{|c|c|c|c|c|c|}
\hline Referência & $\mathrm{n}$ & Sexo & Idade (anos) & $\begin{array}{l}\text { Subestimativa da TMB pelas equações } \\
\text { Schofield (S) ou FAO/WHO/UNU (F) }\end{array}$ & Origem \\
\hline \multirow[t]{2}{*}{ Lawrence et al. (1988) } & 46 & $\mathrm{~F}$ & $20-35$ & $3,0 \%(F)^{1}$ & Escócia \\
\hline & 47 & $\mathrm{~F}$ & $20-35$ & $10,0 \%(F) 1$ & Gâmbia \\
\hline Ferro-Luzzi et al. (1990) & 22 & $\mathrm{~F}$ & $\chi=36$ & $7,0 \%(F)^{2}$ & Etiópia \\
\hline Schultink et al. (1990) & 17 & $\mathrm{~F}$ & $\chi=33$ & $4,0 \%(F)^{1}$ & $\begin{array}{l}\text { República } \\
\text { de Benin }\end{array}$ \\
\hline Spurr et al. (1994) & 21 & $\mathrm{~F}$ & $20-42$ & $0 \%(S)^{2}$ & Colômbia \\
\hline Ategbo et al. (1995) & 34 & $\mathrm{~F}$ & $\chi=31$ & $4,0 \%(F)^{2}$ & $\begin{array}{l}\text { República } \\
\text { de Benin }\end{array}$ \\
\hline \multirow[t]{2}{*}{ Ferro-Luzzi et al. (1997) } & 88 & M & $22-38$ & $0 \%(F)^{2}$ & Índia \\
\hline & 90 & $\mathrm{~F}$ & $22-38$ & $0 \%(F)^{2}$ & \\
\hline
\end{tabular}

Cálculo do \% da subestimativa da TMB: 1 [(TM B medida/TM B estimada) x 100] - 100. 2 não é mencionada forma de cálculo. 
de da coleta de dados de TMB em diferentes grupos populacionais utilizando-se técnicas padronizadas de medição, incluindo estimativas da composição corporal , para que se possa, eventualmente, desenvolver equações a partir de um amplo banco de dados que seja construído com informações adequadas de TMB de várias partes do mundo.

\section{Agradecimentos}

Os autores agradecem ao financiamento parcial do Consel ho Nacional de Desenvolvimento Científico e Tecnológico (Processo 301076/89-8), da Coordenação de Aperfeiçoamento do Pessoal de Ensino Superior (Processo 980466-7), do Fundo de Incentivo à Pesquisa e Eventos do Hospital de Clínicas de Porto Alegre (Processo 990026) e da Fundação de Apoio à Pesquisa do Estado do Rio de Janeiro (Processo E$26 / 150.005 / 2000$ ).

\section{Referências}

ALMEIDA, A. O., 1919. Le métabolisme minimum et le métabolism basal de l'homme tropical de race blanche. Journal de Physiologie et Pathologie Général, 18:713-729.

ALMEIDA, A. O., 1920. Le métabolism basal de I'homme tropical. Journal de Physiologie et PathologieGénéral, 18:958-964.

ALMEIDA, A. O., 1924. L'émission de chaleur le métabolisme basal et le métabolism minimum de I'homme noir tropical. Journal de Physi ologie et Pathologie Général, 22:12-18.

ANJOS, L. A., 1999. Prevalência da inatividade física no Brasil. In: 20 Congresso Brasileiro de Atividade Física e Saúde, Anais, pp. 58-63. Florianópolis: Universidade Federal de Santa Catarina.

ANJOS, L. A.; BRAUN, F. L. C.; DAMIÃO, J. J. \& SILVA, A. F., 1998. Basal metabolic rate cannot be estimated by current predictive equations in Brazilian college female students. Medicine and Science in Sports and Exercise, 30(Sup. 5):S263.

ATEGBO, E. A. D.; VAN RAAIJ, J. M. A.; KONING, F. L. H. A. \& HAUTVAST, J. G. A. J., 1995. Resting metabolic rate and work efficiency of rural Beninese women: A 2-y longitudinal study. American Journal of Clinical Nutrition, 61:466-472.

BANDINI, L. G.; MORELLI, J. A.; MUST, A. \& DIETZ, W. H., 1995. Accuracy of standardized equations for predicting metabolic rate in premenarcheal girls. American Journal of Clinical Nutrition, 62: 711-714.

BENEDICT, F. G., 1928. Basal metabolism data on normal men and women (series II) with some considerations on the use of prediction standards. American Journal of Physiology, 85:607620.

BENEDICT, F. G. \& FINN, M. D., 1928. Normal menstruation and gaseous metabolism. American Journal of Physiology, 86:59-69.
BERKE, E. M.; GARDNER, A. W.; GORAN, M. I. \& POEHLMAN, E. T., 1992. Resting metabolic rate and the influence of pre-testing environment. American Journal of Clinical Nutrition, 55:626629.

BISDEE, J. T.; JAMES, W. P. T. \& SHAW, M. A., 1989. Changes in energy expenditure during the menstrual cycle. British Journal of Nutrition, 61:187199.

BINGHAM, S. A.; GOLDBERG, G. R.; COWARD, W. A.; PRENTICE, A. M. \& CUMMINGS, J. H., 1989. The effect of exercise and improved physical fitness on basal metabolic rate. British Journal of Nutrition, 61:155-173.

BLACK, A. E.; COWARD, W. A.; COLE, T. J. \& PRENTICE, A. M., 1996. Human energy expenditure in affluent societies: An analysis of 574 doubly-labelled water measurements. European Journal of Clinical Nutrition, 50:72-92.

BLUNT, K. \&DYE, M., 1921. Basal metabolism of normal women. Journal of Biological Chemistry, 47: 69-87.

BOOTHY, W. M . \& SANDIFORD, I., 1920. Laboratory Manual of the Technique of Basal Metabolic Rate Determinations. Philadel phia: W. B. Saunders.

BRIEFEL, R. R.; MCDOWELL, M. A.; ALAIMO, K.; CAUGHMAN, C. R.; BISCHOF, A. L.; CARROLL, M. D. \&JOHNSON, C. L., 1995. Total energy intake of the US population: The third National Health and Nutrition Examination Survey, 1988-1991. American Journal of Clinical Nutrition, 62(Sup.):1072S$1080 S$.

BRIEFEL, R. R.; SEMPOS, C. T.; MCDOWELL, M. A.; CHIEN, S. \& ALAIM O, K., 1997. Dietary methods research in the third National Health and Nutrition Examination Survey: Underreporting of energy intake. American Journal of Clinical Nutrition, 65(Sup.):1203S-1209S. 
BROEDER, C. E.; BURRHUS, K. A.; SVANEVIK, L. S. \& WILMORE, J. H., 1992. The effects of either highintensity resistance or endurance training on resting metabolic rate. American Journal of Clinical Nutrition, 55:802-810.

BROZEK, J. \& GRANDE, F., 1955. Body composition and basal metabolism in man: Correlation analysis versus physiological approach. Human Biology, 27:22-32.

BURKE, C. M.; BULLOUGH, R. C. \& MELBY, C. L., 1993. Resting metabolic rate and postprandial thermogenegis by level of aerobic fitness in young women. European Journal of Clinical Nutrition, 47: 575-585.

BURSZTEIN, S.; ELWYN, D. H.; ASKANAZI, J. \& KINNEY, J. M., 1989. Energy Metabolism, Indirect Calorimetry, and Nutrition. Baltimore: Williams \& Wilkins.

CENSI , L.; TOTI , E.; PASTORE, G. \& FERRO-LUZZI. A., 1998. The basal metabolic rate and energy cost of standardized walking of short and tall men. European Journal of Clinical Nutrition, 52:441-446.

CERVATO, A. M.; MAZZILLI, R. N.; MARTINS, I. A. \& MARUCCI, M. F. N., 1997. Dieta habitual e fatores de risco para doenças cardiovasculares. Revista de SaúdePública, 31:227-235.

CLARK, H. D. \& HOFFER, L. J., 1991. Reappraisal of the resting metabolic rate of normal young men. American Journal of Clinical Nutrition, 53:21-26.

CRUZ, C. M.; SILVA, A. F. \& ANJOS, L. A., 1999. A taxa metabólica basal é superestimada pelas equações preditivas em universitárias do Rio de Janeiro, Brasil. Archivos Latinoamericanos de Nutrición, 49:232-237.

CUNNINGHAM, J. J., 1982. Body composition and resting metabolic rate: The myth of feminine metabolism. American Journal of Clinical Nutrition, 36:721-726.

CURTIS, V.; HENRY, C. J. K. \& CHOUEIRI, A. G., 1996. Basal metabolic rate of women on the contraceptive pill. European Journal of Clinical Nutrition, 50:319-322.

DALLOSO, H. M. \& JAMES, W. P. T., 1984. The role of smoking in the regulation of energy balance. International Journal of Obesity and Related Metabolic Disorders, 8:365-375.

DAUNCEY, M. J., 1981. Influence of mild cold on $24 \mathrm{~h}$ energy expenditure, resting metabolism and dietinduced thermogenesis. British Journal of Nutrition, 45:257-267.

DIFFEY, B.; PIERS, L. S.; SOARES, M. J. \& O'DEA, K., 1997. The effect of oral contraceptive agents on basal metabolic rate of young women. British Journal of Nutrition, 77:853-862.

DE BOER, J. O.; VAN ES, A. J. H.; VOORRIPS, L. E.; BLOKSTRA, F. \& VOGT, J. E., 1988. Energy metabolism and requirements in different ethnic groups. European Journal of Clinical Nutrition, 42:983997.

DU BOIS, E. F., 1936. Basal Metabolism in Health and Disease. 3rd Ed. Philadel phia: Lea \& Febiger.

DURNIN, J. V. G. A. \& PASSMORE, R., 1967. Energy, Work, and Leisure. 1st Ed. London: Heinemann Educational Books.

ELLIS, K. J., 1996. Whole body counting and neutron activation analysis. In: Human Body Composition
(A. F. Roche, S. B. Heymsfield \& T. G. Lohman, ed.), pp. 45-61, Champaign: Human Kinetics Books.

FAO (Food and Agriculture Organization)/ WHO (World Health Organization)/UNU (United Nations University), 1985. Energy and Protein Requirements. WHO Technical Report Series 724, Geneva: WHO.

FERRANNINI, E., 1988. The theoretical bases of indirect calorimetry: A review. Metabolism, 37:287301.

FERRO-LUZZI, A.; PETRACCHI, C.; KURIYAN, R. \& KURPAD, A. V., 1997. Basal metabolism of weightstable chronically undernourished men and women: Lack of metabolic adaptation and ethnic differences. American Journal of Clinical Nutrition, 66:1086-1093.

FERRO-LUZZI, A.; SCACCINI, C.; TAFFESE, S.; ABERRA, B. \& DEMEKE, T., 1990. Seasonal energy deficiency in Ethiopian rural women. European Journal of Clinical Nutrition, 44(Sup. 1):7-18.

FIGUEROA-COLON, R.; FRANKLIN, F. A.; GORAN, M. I.; LEE, J.Y. \&WEINSIER, R. L., 1996. Reproducibility of measurement of resting energy expenditure in prepubertal girls. American Journal of Clinical Nutrition, 64:533-536.

GALVÃO, P. E., 1948. Human heat production in relation to body weight and body surface. I. Inapplicability of the surface law on lean men of tropical zone. Journal of Applied Physiology, 1:385-394.

GARROW, J. S., 1974. Energy Bal ance and Obesity in Man. Amsterdam: North-Holland.

GOLDBERG, G. R.; PRENTICE, A. M.; DAVIES, H. L. \& MURGATROYD, P. R., 1990. Residual effect of graded levels of exercise on metabolic rate. European Journal of Clinical Nutrition, 44:99-105.

GREEN, J. H., 1994. Assessment of energy requirements. In: Consensus in Clinical Nutrition. (R. V. Heatley, J. H. Green \& M. S. Losowsky, ed.), pp. 22-37, Cambridge: Cambridge University Press.

HAGGARTY, P.; MCNEILL, G., ABU MANNEH, M. K.; DAVIDSON, L.; MILNE, E.; DUNCAN, G. \& ASHTON, J., 1994. The influence of exercise on the energy requirements of adult males in the UK. British Journal of Nutrition, 72:799-813.

HARRIS, J. A. \& BENEDICT, F. G., 1919. A Biometric Study of Basal Metabolism in Man. Boston: Carnegie Institution of Washington.

HAYTER, J. E. \& HENRY, C. J. K., 1993. Basal metabolic rate in human subjects migrating between tropical and temperate regions: A longitudinal study and a review of previous work. European Journal of Clinical Nutrition, 47:724-734.

HEINI, A. F.; MINGHELLI, G.; DIAZ, E.; PRENTICE, A. M. \& SCHUTZ, Y., 1996. Free living energy expenditure assessed by two different methods in rural Gambian men. European Journal of Clinical Nutrition, 50:284-289.

HENRY, C. J. K. \& EMERY, B., 1986. Effect of spiced food on metabolic rate. Human Nutrition:Clinical Nutrition, 40C:165-168.

HENRY, C. J. K. \& REES, D. G., 1991. New predictive equations for the estimation of basal metabolic rate in tropical peoples. European Journal of Clinical Nutrition, 45:177-185.

HITCHCOCK, F. A. \& WARDWELL, F. R., 1929. Cyclic variations in the basal metabolic rate of women. Journal of Nutrition, 2:203-215. 
JAMES, W. P. T. \& SCHOFIELD, E., 1990. Human Energy Requirements. A Manual for Planners and Nutritionists. Oxford: Oxford University Press.

KENDRICK, Z. V.; MCPEEK C. K. \&YOUNG, K. F., 1990. Prediction of the resting energy expenditure of women following 12 to 18 weeks of very-low-calorie dieting. Annals of Sports Medicine, 5:118-123.

KING, J. T., 1924. Basal Metabolism: Determi nation of the Metabolic Rate in the Practice of Medicine. Baltimore: Williams \& Wilkins.

LAWRENCE, M.; THONGPRASERT, K. \& DURNIN, J. V. G. A., 1988. Between-group differences in basal metabolic rate: An analysis of data collected in Scotland, the Gambia and Thailand. European Journal of Clinical Nutrition, 42:877-891.

LUSK, G., 1917. The Elements of the Science of Nutrition. 3rd Ed. Philadelphia: W. B. Saunders.

MacDIARMID, J. I. \& BLUNDELL, J. E., 1997. Dietary under-reporting: What people say about recording their food intake. European Journal of Clinical Nutrition, 51:199-200.

MCHENRY, E. W. \& BEATON, G. H., 1963. Basic Nutrition. Philadelphia: J. B. Lipincott.

MCNEILL, G.; RIVERS, J. P. W.; PAYNE, P. R.; DE BRITTO, J. J. \& ABEL, R., 1987. Basal metabolic rate of Indian men: No evidence of metabolic adaptation to a low plane of nutrition. Human Nutrition: Clinical Nutrition, 41C:473-483.

MARTINS, I. A.; COELHO, L. T.; MAZZILLI, R. N.; SINGER, J. M.; SOUZA, C. U .; ANTONIETO Jr., A. E.; PASINI, U., NIETO, R. A.; ÁlVARES, E. D. \& OKANI, E. T., 1993. Doenças cardiovasculares ateroscleróticas, dislipidemias, hipertensão, obesidade e diabetes melito em população da área metropolitana da região sudeste do Brasil. I - Metodologia da pesquisa. Revista de Saúde Pública, 27:250-261.

MURGATROYD, P. R.; SHETTY, P. S. \& PRENTICE, A. $M$., 1993. Techniques for the measurement of human energy expenditure: A practical guide. International Journal of Obesity and Related Metabolic Disorders, 17:549-568.

OWEN, O. E.; KAVLE, E.; OWEN, R. S.; POLANSKY, M.; CAPRIO, S.; MOZZOLI, M. A.; KENDRICK, Z. V.; BUSHMAN, M. C. \& BODEN, G., 1986. A reappraisal of caloric requirements in healthy women. American Journal of Clinical Nutrition, 44:1-19.

OWEN, O. E.; HOLUP, J. L.; D'ALESSIO, D. A.; CRAIG, E. S.; POLANSKY, M.; SMALLEY, K. J.; KAVLE, E. C.; BUSHMAN, M. C.; OWEN, L. R.; MOZZOLI, M. A.; KENDRICK, Z. V. \& BODEN, G. H., 1987. A reappraisal of caloric requirements in healthy men. American Journal of Clinical Nutrition, 46: 875-885.

PERKINS, K. A.; EPSTEIN, L. H.; STILLER, R. L.; MARKS, B. L. \&JACOB, R. G., 1989. Acute effects of nicotine on resting metabolic rate in cigarette smokers. American Journal of Clinical Nutrition, 50:545-550.

PIERS, L. S. \& SHETTY, P. S., 1993. Basal metabolic rates of Indian women. European Journal of Clinical Nutrition, 47:586-591.

PIERS, L. S.; SOARES, M. J.; MCCORM ACK, L. M. \& O'DEA, K., 1998. Is there evidence for an age-related reduction in metabolic rate? Journal of Applied Physiology, 85:2196-2204.
PIERS, L. S.; DIGGAVI, S. N.; RIJ SKAM P, J.; VAN RAAIJ, J. M. A.; SHETTY, P. S. \& HAUTVAST, J. G. A. J., 1995. Resting metabolic rate and thermic effect of meal in the follicular and luteal phases of the menstrual cycle in well-nourished Indian women. American Journal of Clinical Nutrition, 61:296302.

PIERS, L. S.; DIFFEY, B.; SOARES, M . J.; FRANDSEN, S. L.; MCCORMACK, L. M.; LUTSCHINI, M.; J. \& O'DEA, K., 1997. The validity of predicting the basal metabolic rate of young Australian men and women. European Journal of Clinical Nutrition, 51:333-337.

POEHLM AN, E. T.; MELBY, C. L. \& BADYLAK, S. F., 1988a. Resting metabolic rate and postprandial thermogenesis in highly trained and untrained males. American Journal of Clinical Nutrition, 47:793-798.

POEHLMAN, E. T.; ARCIERO, P. J.; MELBY, C. L. \& BADYLAK, S. F., 1988b. Resting metabolic rate and postprandial thermogenesis in vegetarians and nonvegetarians. American Journal of Clinical Nutrition, 48:209-213.

PRENTICE, A. M.; BLACK, A. E.; COWARD, W. A. \& COLE, T. J., 1996. Energy expenditure in overweight and obese adults in affluent societies: An analysis of 319 doubly-labelled water measurements. European Journal of Clinical Nutrition, 50:93-97.

RAVUSSIN, E.; BURNAND, B.; SCHUTZ, Y. \&JÉQUIER, E., 1982. Twenty-four-hour energy expenditure and resting metabolic rate in obese, moderately obese, and control subjects. American Journal of Clinical Nutrition, 35:566-573.

REGO, R. A.; BERARDO, F. A. N.; RODRIGUES, S. S. R.; OLIVEIRA, Z. M. A.; OLIVEIRA, M. B.; VASCONCELLOS, C.; AVENTURATO, L. V. O.; MONCAU, J. E. C. \& RAMOS, L. R., 1990. Fatores de risco para doenças crônicas não-transmissíveis: Inquérito domiciliar no município de São Paulo, SP (Brasil). Metodologia e resultados preliminares. Revista de SaúdePública, 24:277-285.

SCHOELLER, D. A., 1999. Recent advances from application of doubly labeled water to measurement of human energy expenditure. Journal of Nutrition, 129:1765-1768.

SCHOFIELD, W. N., 1985. Predicting basal metabolic rate, new standards and review of previous work. Human Nutrition: Clinical Nutrition, 39(Sup. 1): 5-41.

SCHULTINK, W. J.; KLAVER, W.; VAN WIJK, H.; VAN RAAl J, J. M. A. \& HAUTVAST, J. G. A. J., 1990. Body weight changes and basal metabolic rates of rural Beninese women during seasons with different energy intakes. European Journal of Clinical Nutrition, 44(Sup. 1):31-40.

SEALE, J. L. \& RUM PLER, W. V. 1997. Comparison of energy expenditure measurements by diet records, energy intake, doubly labeled water and room calorimetry. European Journal of Clinical Nutrition, 51:856-863.

SEDLOCK, D. A.; FISSINGER, J. A. \& MELBY, C. L., 1989. Effect of exercise intensity and duration on postexercise energy expenditure. Medicine and Science in Sports and Exercise, 21:662-666. SHEPHARD, R. J., 1991. Body Composition in Biologi- 
cal Anthropology. Cambridge: Cambridge University Press.

SHETTY, P. S.; HENRY, C. J. K.; BLACK, A. E. \& PRENTICE, A. M., 1996. Energy requirements of adults: An update on basal metabolic rates (BM Rs) and physical activity levels (PALs). European Journal of Clinical Nutrition, 50(Sup. 1):S11-S23.

SNELL, A. M.; FORD, F. \& ROWNTREE, L. G., 1920. Studies in basal metabolism. Journal of the American Medical Association, 75:515-523.

SOARES, M. J.; FRANCIS, D. G. \& SHETTY, P. S., 1993 Predictive equations for basal metabolic rates of Indian males. European Journal of Clinical Nutrition, 47:389-394.

SOARES, M. J.; SEQUEIRA, J. \& SHETTY, P. S., 1988. The effect of the preceding day's protein intake on basal metabolic rates in young adults. British Journal of Nutrition, 60:425-431.

SOARES, M. J. \& SHETTY, P. S., 1988. Validity of Schofield's predictive equations for basal metabolic rates in Indians. Indian Journal of Medical Research, 88:253-260.

SOLOMON, S. J.; KURZER, M. S. \& CALLOWAY, S. H., 1982. Menstrual cycle and basal metabolic rate in women. American Journal of Clinical Nutrition, 36:611-616.

SPURR, G. B.; DUFOUR, D. L.; REINA, J. C.; HOFFMANN, R. G.; WASLIEN, C. I. \& STATEN, L. K., 1994. Variation of the basal metabolic rate and dietary energy intake of Colombian women during 1 y. American Journal of Clinical Nutrition, 59:2027.

TAI, M. M.; CASTILLO, P. F. \& SUNYER, F. X. P., 1997. Thermic effect of food during each phase of the menstrual cycle. American Journal of Clini cal Nutrition, 66:1110-1115.

TAYLOR, M. C.; MacLEOD, G. \& ROSE, M. S., 1956. Foundations of Nutrition. 5th Ed. New York: Macmillan Company.

TURLEY, K. R.; MCBRIDE, P. J. \& WILMORE, J. H., 1993. Resting metabolic rate measured after subjects spent the night at home vs. at a clinic. American Journal of Clinical Nutrition, 58:141-144.

TZANKOFF, S. P. \& NORRIS, A. H., 1977. Effect of muscle mass decrease on age-related BMR changes. Journal of Applied Physi ology: Respiratory, Environmental and Exercise Physiology, 43:1001-1006.

TZANKOFF, S. P. \& NORRIS, A. H., 1978. Longitudinal changes in basal metabolism in man. Journal of Applied Physi ology: Respiratory, Environmental and Exercise Physiology, 45:536-539.
VALENCIA, M. E.; M OYA, S. Y.; M cNEI LL, G. \& HAGGARTY, P., 1994. Basal metabolic rate and body fatness of adult men in northern Mexico. European Journal of Clinical Nutrition, 48:205-211.

VINKEN, A. G.; BATHALON, G. P.; SAWAYA, A. L.; DALLAL, G. E.; TUCKER, K. L. \& ROBERTS, S. B., 1999. Equations for predicting the energy requirements of healthy adults aged 18-81 years. American Journal of Clinical Nutrition, 69:920-926.

WACK, J.T. \& RODIN, J., 1982. Smoking and its effects on body weight and the systems of caloric regulation. American Journal of Clinical Nutrition, 35: 366-380.

WAHRLICH, V., 2000. Taxa Metabólica Basal em MuIheres Resi dentes em Porto Al egre, Rio Grande do Sul. Dissertação de Mestrado, Rio de Janeiro: Instituto de Nutrição, Universidade Federal do Rio deJaneiro.

WAHRLICH, V. \& ANJ OS, L. A., 2000. Basal metabolic rate of young women living in tropical and temperate regions of Brazil. Medicine and Science in Sports and Exercise, 32(Sup. 5):S172.

WARWICK, P. M. \& BUSBY, R., 1993. Prediction of twenty-four-hour energy expenditure in a respiration chamber in smokers and non-smokers. European Journal of Clinical Nutrition, 47:600603.

WARWICK, P. M.; EDMUNDSON, H. M. \& THOMPSON, E. S., 1995. No evidence for a chronic effect of smoking on energy expenditure. International Journal of Obesity and Related Metabolic Disorders, 19:198-201.

WEBB, P., 1985. Human Calorimeters. New York: Praeger Scientific.

WEIR, J. B., 1949. New methods for calculating metabolic rate with special reference to protein metabolism. Journal of Physiology, 109:1-9.

WESTRATE, J. A.; WEYS, P.; POORTVLIET, E.; DEURENBERG, P. \& HAUTVAST, J. G. A. J., 1990. Lack of a systematic sustained effect of prolonged exercise bouts on resting metabolic rate in fasting subjects. European Journal of Clinical Nutrition, 44:91-97.

WILMORE, J. H.; STANFORTH, P. R.; HUDSPETH, L. A.; GAGNON, J.; DAW, E. W.; LEON, A. S.; RAO, D. C.; SKINNER, J. S. \& BOUCHARD, C., 1998. Alterations in resting metabolic rate as a consequence of 20 wk of endurance training: The Heritage Family Study. American Journal of Clinical Nutrition, 68:66-71.

WILTSHIRE, M. O. P., 1921. Some observations on basal metabolism in menstruation. Lancet, 2:388389. 